\title{
ELECTRICAL AND SPECTROSCOPIC STUDIES OF THE EFFECTS OF DC ELECTRIC FIELDS ON PRE-MIXED PROPANE-AIR FLAMES
}

\author{
S. D. Marcum \\ Department of Physics, Miami University \\ Oxford, OH 45056 \\ G. D. Gillen \\ Department of Physics, The Ohio State University \\ Columbus, $\mathrm{OH} 43210$ \\ B. N. Ganguly \\ Air Force Research Laboratory \\ Wright-Patterson AFB, OH 45433
}

\begin{abstract}
$\underline{\text { Abstract }}$
The effects of DC electric fields on temperature distributions within pre-mixed propane-air flames have been measured by the technique of thin filament pyrometry (TFP). We have focussed on the dramatic electric-field-induced modifications of the shape and size of the inner cone and the concomitant changes in the temperature profiles of fuel-rich mixtures with equivalence ratios of 1-1.35. Temperature profile measurements show large decreases in the reaction zone volume that is dependent upon the applied voltage polarity, indicating that electron impact excitation is not responsible for the observed effects. Additionally, the observed flame temperature profile modifications are not strongly dependent on the flow velocity. The TFP results have been verified by spectroscopic measurements of the rotational temperature of the $\mathrm{CH}\left(\mathrm{A}^{2} \Delta-\mathrm{X}^{2} \Pi\right)$ emission band at $431 \mathrm{~nm}$. Due to the better coupling of the field to the flame in the present experiments, the magnitude of the externally applied voltage necessary to
\end{abstract}

produce large changes in inner cone geometry are substantially smaller than those used in previous experiments. Electrical measurements that have been made include vertical and horizontal variations of the floating potential within the flames, and bulk current and voltage characteristics. High-speed imaging of the flame response to pulsed DC voltage has been performed to investigate the dynamics of the ionic wind effects. All measurements are consistent with the observed flame perturbations being a fluid mechanical response to the applied field.

\section{$\underline{\text { Introduction }}$}

A rather large amount of work has been reported on the various effects of electric fields on a wide variety of flames ${ }^{1-10}$. However, the mechanism(s) responsible for the rather dramatic field-induced effects on the size and shape of the inner cone of some hydrocarbon flames is still not well understood. Bradley ${ }^{1}$ provides a good overview of the subject, as well 
as a thorough review of the literature in this general area up to the date of that text's publication.

Electric-field-induced modification of flame geometry has been known for some time, the first report being published by Chattock $^{2}$ in 1899. In more recent studies, many aspects of the effects of externally applied fields on flames have been investigated. Increases in blowoff flow rates due to external fields in both diffusion $^{3}$ and premixed ${ }^{4}$ methane-air flames have been measured. Similar increases in flame stability have also been observed at fields sufficient to produce a corona discharge ${ }^{5}$ within the flame. Improved heat transfer to solid surfaces ${ }^{6}$ due to externally applied fields has been reported. Increased burning velocities for hydrocarbon flames in DC fields ${ }^{7}$ and elevated electron temperatures for flames in microwave fields ${ }^{8}$ have been similarly established. More recently, a reaction kinetics model ${ }^{11}$ simulating the effects of electric fields in premixed methane flames has been published, and electric fields have been used to counterbalance natural convection and simulate microgravity ${ }^{12}$ in small diffusion flames.

The present study reports non-intrusive measurements of DC electric-field-induced changes in the temperature profiles of hydrocarbon flames using thin filament pyrometry. Spectroscopic measurements of the $\mathrm{CH}(\mathrm{A}-\mathrm{X})$ bands confirms the TFP results. Floating potentials within the flames have been measured in a more intrusive way. The temporal characteristics of the flame's response to a pulsed voltage are also documented. Together they provide insight into the processes responsible for the field-induced flow known as the Chattock (ionic) wind that is responsible for the flame geometry changes studied here.

\section{ExperimentalApparatus and Procedures}

The apparatus used to make spatially resolved temperature measurements within the flame as functions of applied voltage, polarity, fuel-to-air ratio and flow rate is shown schematically in Figure 1. The burner used was a commercially available atomic absorption type (Perkin-Elmer) with a modified, 17-mm diameter head consisting of multiple layers of fine-mesh stainless steel in a brass housing. DC fields were applied to the flames by connecting a power supply $(1000-3000 \mathrm{~V})$ across the burner head and an electrode centered between 30-40 $\mathrm{mm}$ above the grounded burner.

In early experiments, the electrode was a 25-mm diameter stainless steel ring. However, it was found that much better electrical coupling to the flame was achieved by covering the ring electrode with a 1-mm grid stainless steel screen. The bulk of the results reported here were derived from the latter electrode geometry.

Commercially available silicon carbide fibers nominally $15 \mathrm{um}$ in diameter were suspended under slight tension horizontally across the flame. The glowing fiber was imaged onto a filter detector combination using a 30-cm focal length, 75-mm diameter lens. The filter used was a long-pass type that transmitted wavelengths greater than $780 \mathrm{~nm}$, and was used to prevent bright visible flame emissions from reaching the detector. The detector was a silicon PIN diode with a $1 \mathrm{~mm}^{2}$ active area and the detector current was measured by an electrometer working in the current mode. The nano-ammeter was in turn interfaced to a PC that was used for experimental control and data reduction.

The burner was mounted on translation stages so that temperature measurements at different locations within the flame could be accomplished simply by repositioning the burner. Temperature measurements were made along the length of the SiC fiber at 1-mm intervals. The glowing fiber was observed at a number of locations along its length sufficient to span the width of the flame. Vertical translation of the burner allowed horizontal temperature profile measurements to be made at different distances above the burner head. Such temperature profile measurements were performed at 3-mm intervals starting $3 \mathrm{~mm}$ above the burner head and continuing to locations above the peak of the inner cone. The 
total number of horizontal profiles measured for each flow condition studied depended on the height of the inner cone.

For most flow conditions studied, the propane flow rate was held constant at 590 $\mathrm{sccm}$. The air flow rate was varied from a low of $6580 \mathrm{sccm}$ to that which provided stoichiometric conditions. Results from flames with equivalence ratios between 1.2 and 1.4 are reported here. In order to gauge the dependence of the electric-field-induced modification of such flames on flow velocity, one data set was recorded for an equivalence ratio of 1.35 using a 50 percent higher overall flow rate than that cited above. The relevant details of the thin filament pyrometry technique are presented below.

Floating potential measurements were made along the axis of the flame, as well horizontally as across the flame at various heights above the burner. The voltage probe consisted of a $0.75-\mathrm{mm}$ diameter chromel wire housed in 1-mm inner diameter aluminum oxide tube. The length of the wire protruding through one end of the insulating housing and exposed to the electrically biased flame was $1 \mathrm{~mm}$. Floating potentials were measured at $1-\mathrm{mm}$ intervals both horizontally and vertically. However, the relatively large size of the voltage probe disturbed the flame flow field somewhat, especially near the surface of the inner cone. Nevertheless, the general features of the horizontal and vertical floating potential profiles were recorded. Additionally, current/voltage characteristics were measured for each flow condition studied.

Spectroscopic measurements were performed using a CCD-based spectroscopy system (Photometrics Model CCD9000) mounted on a $0.27-\mathrm{m}$ spectrometer (Jarrel-Ash MonoSpec 27). The spectrometer has three gratings mounted on a rotatable turret, which allows rapid variation of the system dispersion.

High-speed 2-dimensional images used to document the flame's dynamic response to a pulsed DC bias were made using a intensified, gateable CCD camera (Princeton Instruments Model ICCD-576). One-hundred microsecond exposures spaced $1 \mathrm{~ms}$ apart were recorded after the rise and fall of the pulsed-DC field.

\section{$\underline{\text { Thin Filament Pyrometry }}$}

TFP provides a low-cost method of measuring temperatures in combusting flows without the significant flow field perturbations caused by thermocouples or the cost and complexity associated with laser-based temperature diagnostics ${ }^{13}$. This work represents the first application of TFP to measurements of temperatures within flames under electrical stress.

TFP is based upon measurements of the blackbody emission of a fine silicon carbide filament (nominally 15 um diameter) that is suspended across the flame. SiC has a constant, high emissivity (0.88) over the temperature range of interest in this study ${ }^{13}(1000-2200 \mathrm{~K})$. That material also has a sufficiently low thermal conductivity to allow emission to be measured at selected points along its length, thus providing for spatially resolved radial temperature measurements. $\mathrm{SiC}$ fibers of the type used in this study are commercially available. The emission intensity of the filament is given by Planck's blackbody radiation formula multiplied by the emissivity,

$$
I(\lambda, T)=\frac{\varepsilon(\lambda, T) C_{1}}{\lambda^{5}\left(\exp \left(C_{2} / T\right)-1\right)}
$$

where $\varepsilon(\lambda, T)$ is the filament emissivity, $C_{1}$ and $C_{2}$ are the radiation constants, $\lambda$ is wavelength and $T$ is the filament temperature. Following Goss, et. al., ${ }^{13}$ the detector signal can be represented by the following relation,

$$
S_{\text {exp }}=k_{\exp } \int I(\lambda, T) R(\lambda) \operatorname{Tr}(\lambda) d \lambda
$$

Here, $\operatorname{Tr}(\lambda)$ represents the transmission characteristics of the optics used, $R(\lambda)$ is the detector response and $k_{\text {exp }}$ is an experimental constant which includes the optical collection efficiency and the gain of the detector electronics. The latter constant is removed in practice by normalizing the observed signal to the signal at a known temperature (i.e. at 
stoichiometric conditions). Numerical integration of equation 2 for the apparatus used for this study yields the filament temperature calibration plot shown in Figure 2. The filament temperature can thus be extracted from Figure 2 after measurement of the detector current for a known temperature condition, $I_{\text {cal }}$, by comparison to the current measured under experimental conditions, $I_{\text {exp }}$.

To reduce the measured filament temperature to that of the surrounding gas, corrections for convective and radiative losses must be made. ${ }^{13}$ The necessary heat balance analysis for the experiment described here has been applied to the results of the numerical integration of equation 2 and incorporated into the temperature calibration plot of Figure 2.

\section{$\underline{\text { Results and Discussion }}$}

\section{$\underline{\text { Temperature Profile Measurements }}$}

All experimental results presented here are derived from experiments that used a grounded burner and a positively biased upper electrode. The horizontal temperature profiles measured by the procedure describe above for the case of the equivalence ratio of 1.35 are shown in Figure 3 for the screen anode geometry. In that Figure, results are shown for the normal flame (no applied field) by solid lines and for the field-modified flame by dashed lines. The total voltage drop across the flame was $2500 \mathrm{~V}$. The vertical lines shown at each data point location are $\pm 80 \mathrm{~K}$ error bars.

The horizontal profiles in the top-most plot of Figure 3 are for a location just above the peak of the inner cone in the unperturbed flame. It is clear that the effect of the applied voltage on this portion of the flame is negligible. Temperature profiles measured at locations successively closer to the burner head show markedly different field-induced effects. Between 18 and $6 \mathrm{~mm}$ above the burner head, the horizontal temperature profiles with and without an applied field are radically different. The temperature profile measured at $3 \mathrm{~mm}$ above the burner head again shows only slight differences between the applied field and no applied field cases.

A general comment is in order at this point. As the applied field is increased, the reduction of the inner cone height shown in Figure 3 is accompanied by increased turbulence. Voltage probe measurements also show increased voltage amplitude fluctuations at frequencies higher than natural flame flicker frequencies. The surface of the inner "cone" has its shape altered dramatically by the applied field and the surface oscillates at essentially the same frequency observed with the voltage probe. The depressed inner "cone" typically appears as multiple, oscillating small cones. Additionally, the depressed inner "cone's" surface was observed to substantially alter its shape occasionally, and oscillate about a new quasi-stable shape, without any noticeable perturbation that could be taken as the cause for the change. It was as if the oscillations of the inner cone were chaotic in some sense. Such changes in overall inner cone shape occurred between data recording for the horizontal profiles at 12,9 and $6 \mathrm{~mm}$ above the burner head. Those changes are reflected in the temperature profiles for those locations that appear in Figure 3.

For the same fuel/air flow conditions as those just discussed, similar field-induced effects on the temperature profiles are found for the ring anode geometry described in the previous section. However, the ring anode geometry required a $2500 \mathrm{~V}$ bias for the production of the same general level of fieldinduced flame perturbations shown in Figure 3, which used only a $1500 \mathrm{~V}$ total drop across the flame. The reduction in applied voltage necessary to produce a given modification of the reaction zone with the screen anode is easily understood in terms of the much better coupling of the electric field between the screen electrode and the flame. Producing the same field-induced flame perturbations with a lower voltage would have obvious advantages in any practical application of the effects described here. 
In order to investigate the dependence of the field induced reaction zone modifications on flow velocity, temperature profiles were measured for a flame with the same equivalence ratio as that used in the previous two cases, but with a $50 \%$ increase in overall flow rate. Results for this high flow case are shown in Figure 4. Comparison of Figures 3 and 4 indicate that the field-induced flame temperature profile modifications are not strongly dependent on the gas flow velocity. The dramatic compression of the inner cone toward the burner head when an electric field was applied occurred to varying degrees over the entire range of equivalence ratios studied (1.0 - 1.57).

It is significant to note that none of the field-induced temperature profile modifications described above occur for any flame condition investigated if the polarity of the applied voltage is reversed. Additionally, other than relocation of the flame front, no measurable difference in flame temperature was observed either at, or outside the surface of the inner cone. CARS temperature measurements were also performed with and without an applied voltage at locations just above the tip of the unperturbed reaction zone for each case described above. All CARS-based temperature measurements agreed with the TFP measurements within experimental error.

\section{$\underline{\text { Electrical Measurements }}$}

Horizontal and vertical floating potential profiles were also measured for each flow conditions and electrode geometry described above. Figure 5 shows such floating potential profiles for an equivalence ratio of 1.35 and the ring anode geometry. Such profiles are typical of those found with the ring anode geometry and with other equivalence ratios.

The upper plot in Figure 5 shows the vertical floating potential profile measured along the flame axis at locations separated by 1 $\mathrm{mm}$ for an overall bias of $1500 \mathrm{~V}$. As indicated, the bulk of the voltage drop occurs near the anode. More significantly, the slope of the floating potential profile slope changes sign near the applied-field-modified surface of the reaction zone. The lower plot in Figure 5 shows the measured horizontal floating potential profiles at two locations above the burner head. At $21 \mathrm{~mm}$ above the burner head, well above the field-modified reaction zone, the floating potential profile is flat, indicating a relatively small electric field in that region. However, the profile at $15 \mathrm{~mm}$ above the burner head, near the surface of the modified reaction zone, shows a much larger floating potential gradient (electric field) that is directed toward the burner head and inward toward the flame axis.

The final electrical measurements presented here are the current/voltage characteristics shown in Figure 6. Those I/V characteristics compare the ring and screen anode geometries, and were measured under the same conditions as for the floating potential measurements just described. The significant features of Figure 6 is the very small amount of electrical input power required to produce the rather significant field-induced flame temperature modifications described above, and the much better coupling of the external circuit to the flame with the screen anode. The largest current drawn for any flow condition studied was less than 300 microamperes for the largest bias of $3000 \mathrm{~V}$, or less than $1 \mathrm{~W}$ of input electrical power. That level of input power is negligible in comparison to the rate of chemical energy release in the flame.

\section{Spectroscopic Measurements}

The spectroscopic measurements were primarily intended to provide another independent check on the TFP temperature measurements. However, another interesting result of the spectroscopic study was also observed. The $\mathrm{CH}$ radical emission as a function of equivalence ratio strongly correlates with the analogous trend for the allowed current through the flame at fixed bias, as shown in Figure 7. Gaydon and Wolfhard ${ }^{14}$ note that there is a similarity in the occurrence of $\mathrm{CH}$ 
emission in flames and chemi-ionization suggesting that $\mathrm{CH}$ ground state molecules and excited radicals may play a significant role in ion production in hydrocarbon flames. The results shown in Figure 7 support that view, given that the flame conductivity peaks very near the equivalence ratio that provides maximum $\mathrm{CH}^{*}$ emission intensity.

High resolution spectra of the $\mathrm{CH} 431$ $\mathrm{nm}$ band and the resulting Boltzmann plot are shown in Figure 8. Rotational temperatures determined from the distribution of intensities over the rotational structure of the $\mathrm{CH}(\mathrm{A}-\mathrm{X})$ band showed no change when an external field was applied. The rotational temperature measured for all cases was essentially equal to the gas temperature at a given equivalence ratio. In addition to indicating that $\mathrm{CH}(\mathrm{A}-\mathrm{X})$ rotational temperature measurements also provide a good indicator of the temperature of electrically stressed propane flames, the results also provide an independent verification of the TFP measurements. For the case shown in Figure $8(\phi=1.2)$, the rotational temperature was $2040 \mathrm{~K} \pm 50 \mathrm{~K}$.

\section{High-Speed Two-Dimensional Imaging}

The final experimental results reported here is the flame response to a $2.1 \mathrm{kV}$ square voltage pulse. Figure 9 shows two sets of individual $100 \mu$ s exposures of the flame's inner cone spaced $1 \mathrm{~ms}$ apart after the rise (top set) and fall (bottom set) of the bias pulse for an equivalence ratio. The rise time of the voltage pulse was less than $0.4 \mathrm{~ms}$, and the fall time was in the 10's of microseconds range. As shown, the time evolution of the geometry of the reaction zone agrees well with the results of the floating probe measurements, which indicated that the electric field points inward toward the flame axis and downward toward the grounded burner head.

The time scale of the flame response is $\sim 10 \mathrm{~ms}$ for both rise and fall of the pulsed bias voltage. The ionic wind increases the effective dynamic pressure on the flame front, causing it to collapse inward toward the flame axis and then downward along the axis toward the burner head. . The imaging results suggest that the effective fluid mechanical response of the flame is altered by the applied field.

\section{$\underline{\text { Conclusions }}$}

Although there is wide opinion in the literature $^{1-10}$ as to the causes of similar effects in flames subjected to electric fields, we feel that probable mechanisms for the above described are heavy charged particle induced momentum transfer effects such as the ion drag force. ${ }^{15}$ Draine and Salpeter ${ }^{12}$ write an expression for the ion drag force that depends strongly on the net charge of the particles and their velocity. The overall role of the ion drag force in sooty (or dusty) plasmas using screened Coulomb force to describe the collisions with particles has been investigated ${ }^{16}$. The magnitudes of the resulting cross sections can be very large at low ion velocities.

The ionic wind does not perturb the flame chemistry, although mixing rates are clearly affected. The net result of the fielddirected force on the flame ions is to increase the effective dynamic pressure on the flame front. The temporal response of the flame to a pulsed bias voltage shows that the reaction zone modification is a fluidics effect. It is obvious that whatever process produces the observed reaction zone modifications also produces turbulence. The fact that reversal of the applied voltage polarity produces no observable changes with DC bias indicates that electron impact excitation cannot be responsible for the observed reaction zone modifications.

\section{Acknowledgements}

The authors would like to thank Dr. Larry Goss and Mr. Michael Post for their valuable advice on the TFP techniques and their assistance in developing the filament temperature calibration. Additional thanks to 
Dr. Goss for performing the CARS temperature verification measurement.

\section{$\underline{\text { References }}$}

1. Bradley, D., in Advanced Combustion Methods, F.J. Weinberg, Ed., Academic Press, New York (1986)

2. Chattock, A.P., Phil. Mag 48, 401 (1899).

3. Calcote, H.F. and Pease, R.N., Ind. Eng. Chem. 43, 2726 (1951).

4. Calcote, H.F. and Berman, C.H., in Fossil Fuels Combustion Symposium PD-Vol. 25, S.N. Singh, Ed., 25 (1989).

5. Bradley, D. and Nasser, S.H., Combust. Flame 55, 53 (1984).

6. Bradley, D. and Ibrahim, S.M.A., $15^{\text {th }}$ Symposium (International) on Combustion, The Combustion Institute, Pittsburgh, PA, p 1023.

7. Jaggers, H.C. and von Engel, A., Combust. Flame 16, 275 (1971).

8. Maclatchy, D.S., Clements, R.M. and Smy, P.R., Combust. Flame 45, 161 (1982).
9. Kono, M., Carleton, F.B., Jones, A.R. and Weinberg, F.J., Combust. Flame 78, 357 (1989).

10. Gulyaev, G.A., Popkov, G.A. and Shebeko, Yu.N., Phys. Combust. Explosion 21, 25 (1985).

11. Pedersen, T. and Brown, R.C., Combust. Flame 94, 433 (1993).

12. Strayer, B.A., Posner, J.D., Dunn-Rankin, D. and Weinberg, F.J., Proc. R. Soc. Lond. A, Math. Phys. Eng. Sci. 458, 1151 (2002).

13. Goss, L.P., Vilimpoc, V., Sarka, B and Lynn, W.F., Jour. Engr. Gas Turb. Pwr. 111, 46 (1989).

14. Gaydon, A.G. and Wolfhard, H.G., Flames: Their Structure, Radiation and Temperature, $4^{\text {th }}$ Edition, Chapman and Hall, London (1979), p 359.

15. Draine, B.T. and Salpeter, E.E., Astrophys. J. 231, 77 (1979).

16. Kilgore M.D., Daughtery J. E., Porteous R.K., and Graves, D.B., J. Appl. Phys.73, 7195 (1993). 
Figure 1. Experimental setup.

Figure 2. Flame temperature calibration plot from numerical integration of Eq. (2). Radiative and convective corrections have been applied.

Figure 3. Horizontal flame temperature profiles measured for the screen anode geometry.

Figure 4. Horizontal flame temperature profiles measured at $50 \%$ higher overall flow rate than for those shown in Figure 3.

Figure 5. Typical vertical (upper plot) and horizontal floating potential profiles.

Figure 6. Typical current/voltage characteristic measured for field-modified flames. Note that the total electrical power input is quite small $(<1 \mathrm{~W}$ at the highest bias).

Figure 7. Allowed current and $431 \mathrm{~nm}$ band intensities as a function of equivalence ratio.

Figure 8. High resolution spectrum of the CH $431 \mathrm{~nm}$ band and associated Boltzmann plot.

Figure 9. High-speed two-dimensional images $(100 \mu$ s exposures $)$ of the flame response to a pulsed bias.
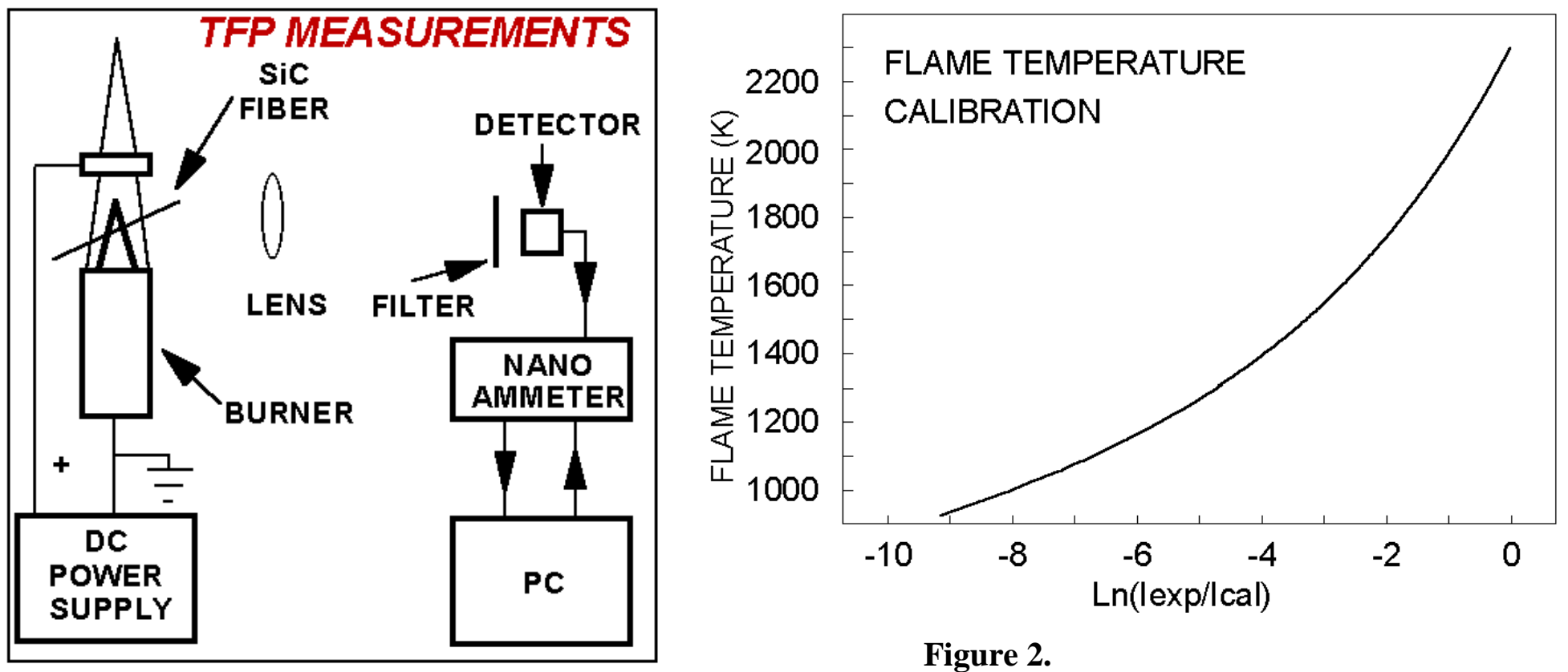

Figure 2.

Figure 1. 


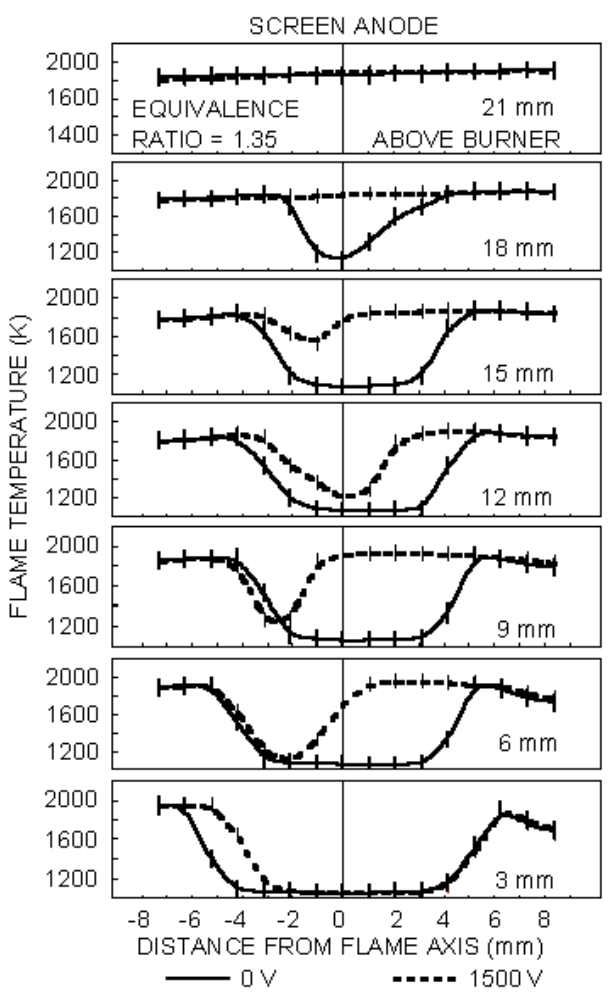

Figure 3 .

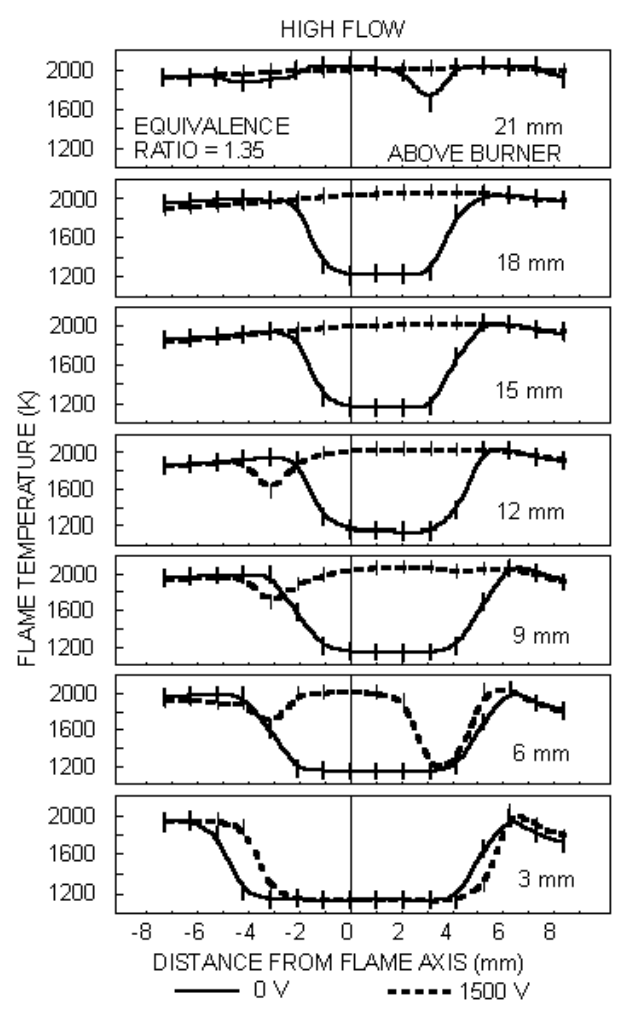

Figure 4.
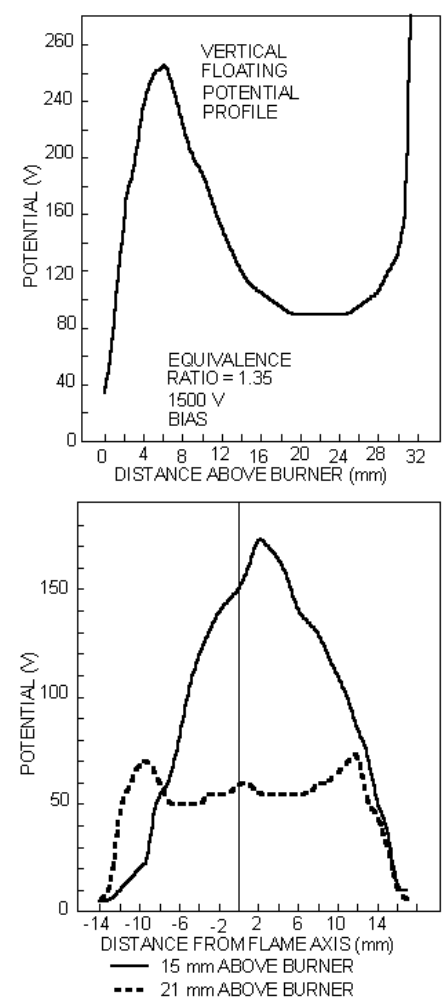

Figure 5.

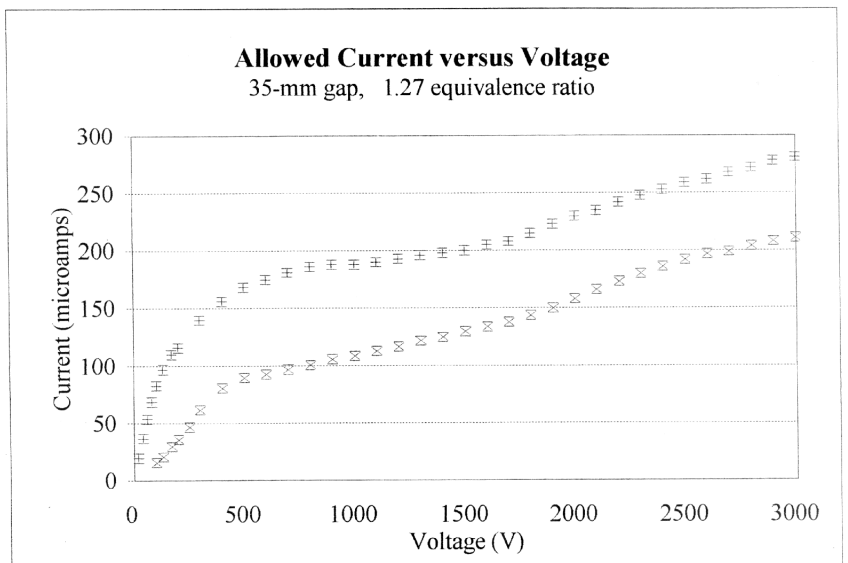

Figure 6.
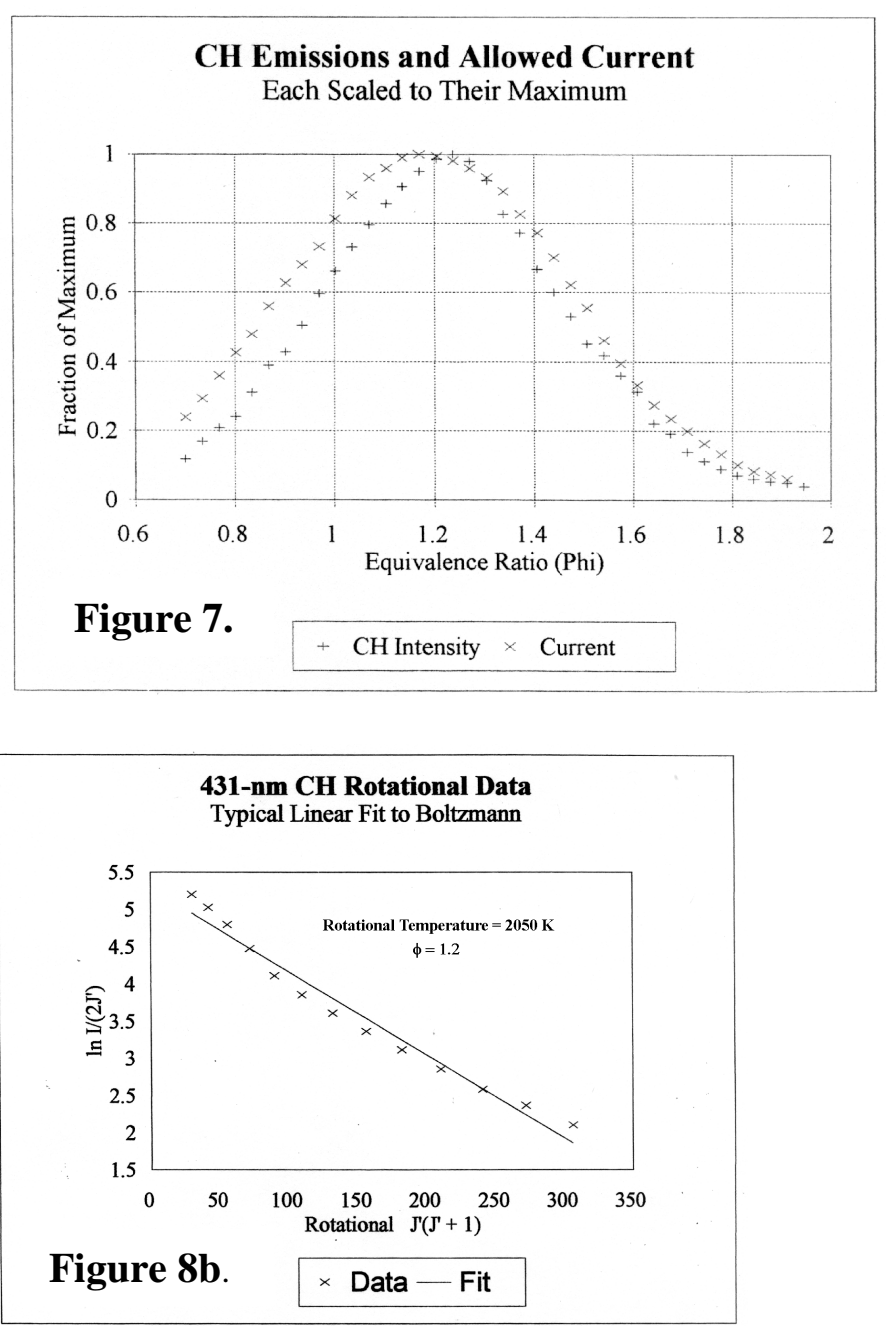


\section{Voltage rise}
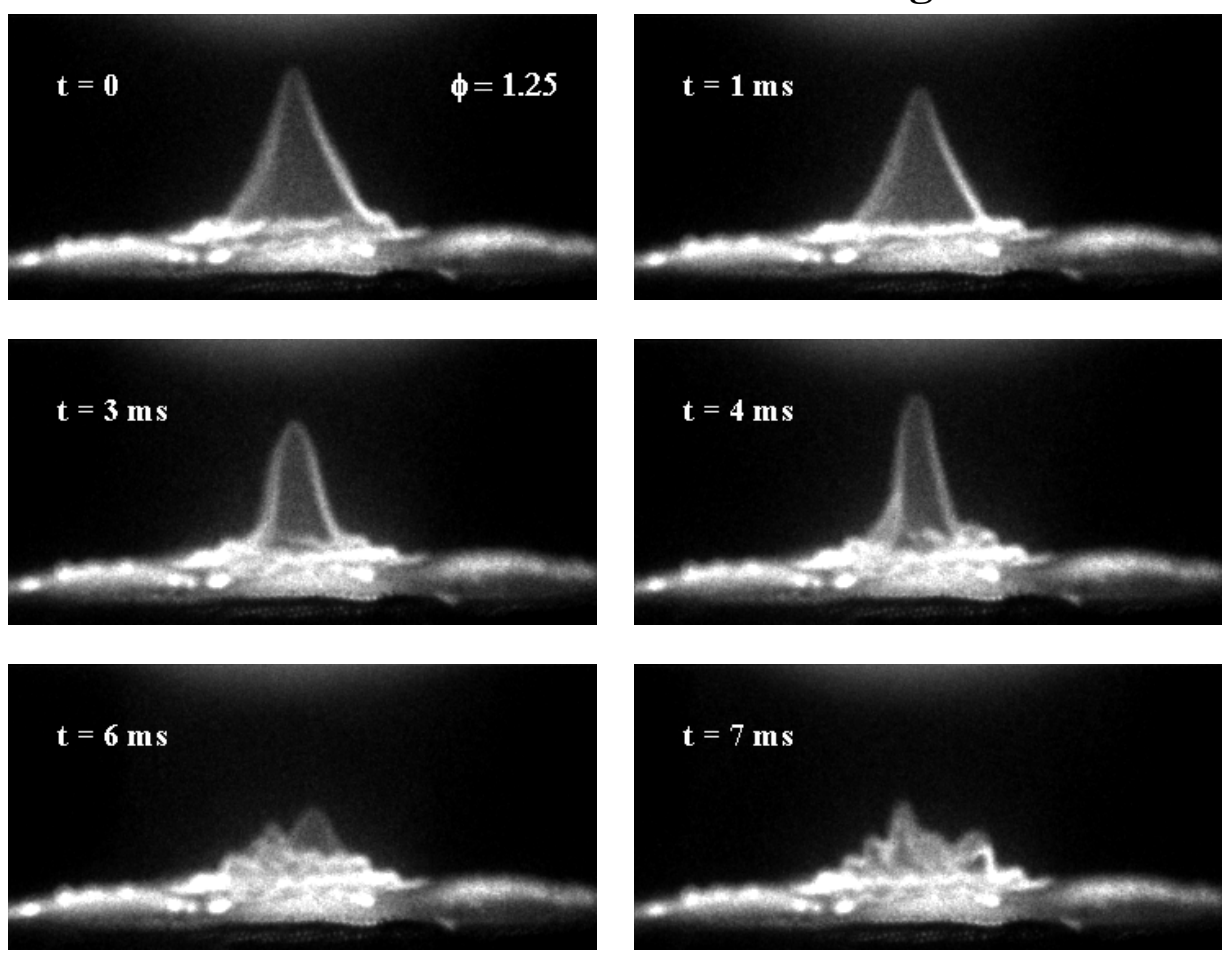

\section{Voltage fall}
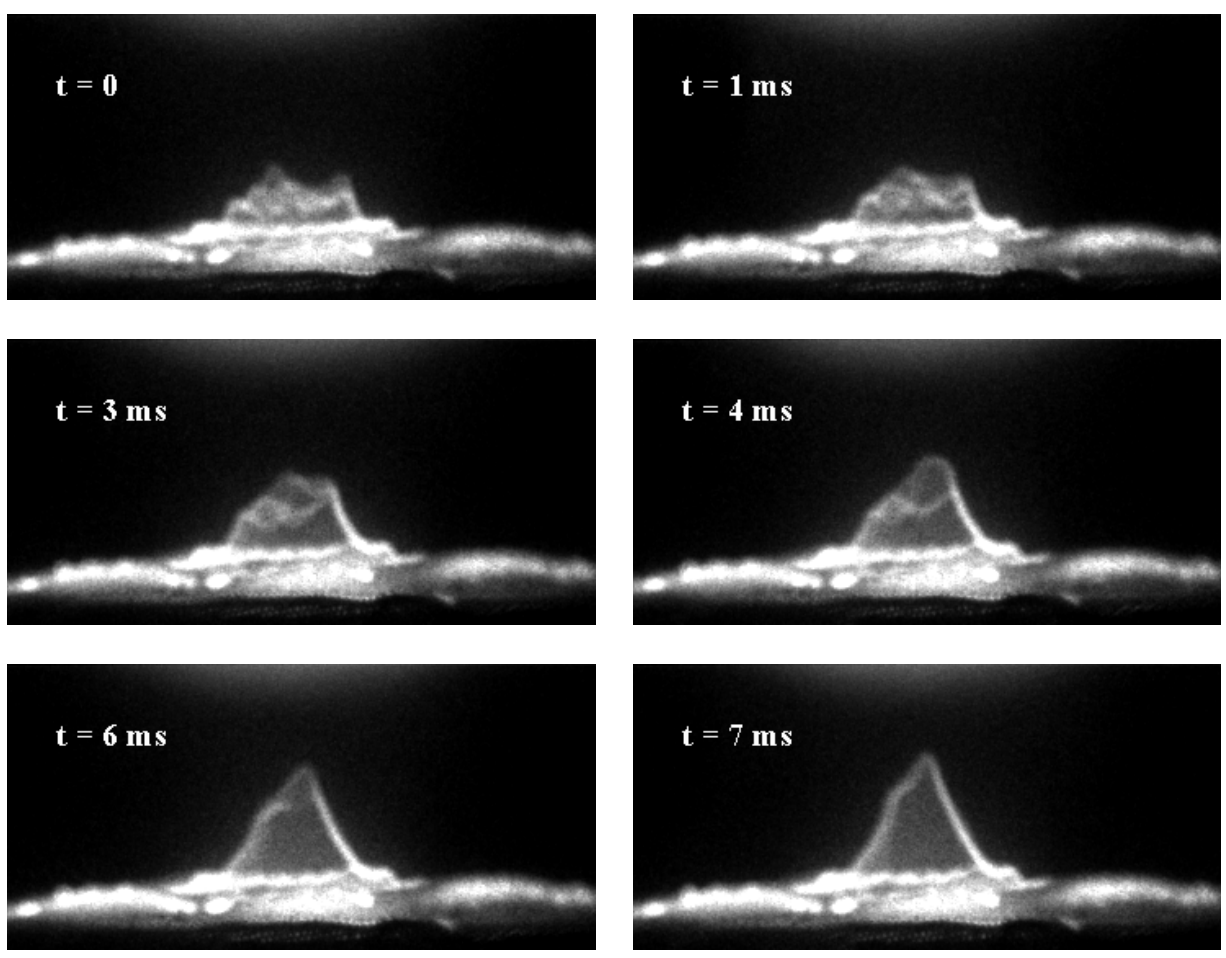
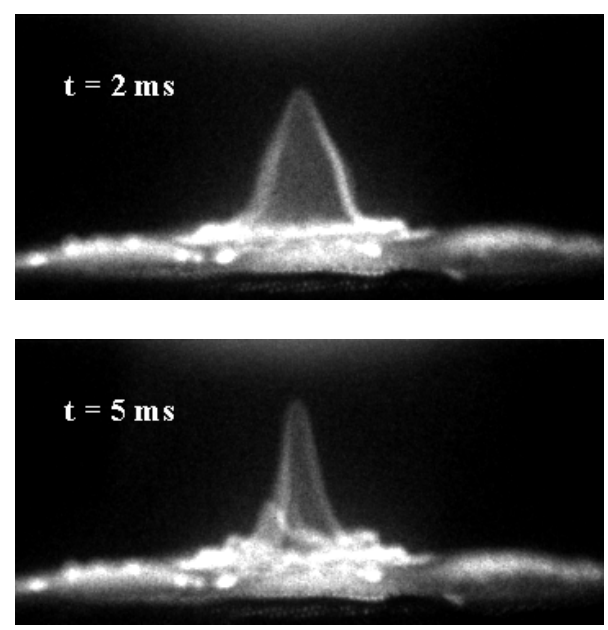

$\mathrm{t}=8 \mathrm{~ms}$

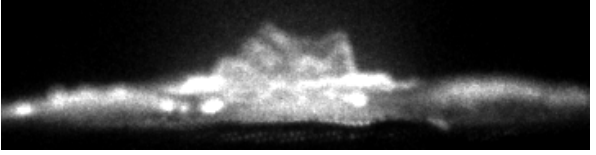

$\mathrm{t}=2 \mathrm{~ms}$
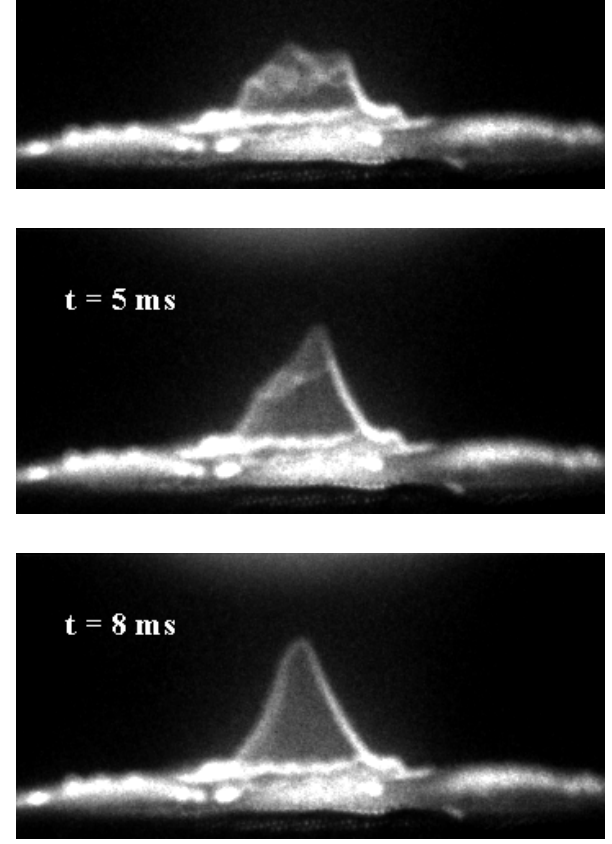

Figure 9. 